\title{
Gastroenterologists' Views of Shared Decision Making for Patients with Inflammatory Bowel Disease
}

\author{
Corey A. Siegel ${ }^{1} \cdot$ Jennifer H. Lofland ${ }^{2} \cdot$ Ahmad Naim $^{2,3} \cdot$ Jan Gollins $^{4} \cdot$ \\ Danielle M. Walls ${ }^{5}$ Laura E. Rudder ${ }^{6}$. Chuck Reynolds ${ }^{6}$
}

Received: 24 October 2014/Accepted: 17 April 2015/Published online: 5 May 2015

(C) The Author(s) 2015. This article is published with open access at Springerlink.com

\begin{abstract}
Background There is limited information on gastroenterologists' perspectives of shared decision making (SDM) in discussions of therapeutic agents with inflammatory bowel disease (IBD) patients.

Aims To examine gastroenterologists' perspectives about SDM with IBD patients, using a novel statistical hybrid approach to analyze qualitative data.

Methods Physician interviews and online surveys were conducted from a panel of gastroenterologists in April 2012. Gastroenterologists were asked about their barriers to SDM, SDM practices, relationship to their patients, knowledge of SDM, and insights into SDM implementation. Key audio
\end{abstract}

These data have been presented in part at the American College of Gastroenterology Annual Meeting on October 22, 2012, in Las Vegas, NV.

Electronic supplementary material The online version of this article (doi:10.1007/s10620-015-3675-z) contains supplementary material, which is available to authorized users.

Jennifer H. Lofland

jlofland@its.jnj.com

Corey A. Siegel

corey.a.siegel@hitchcock.org

Ahmad Naim

anaim@incyte.com

Jan Gollins

jan@deltamodelling.com

Danielle M. Walls

danielle@bdjsolutions.com

Laura E. Rudder

laurar@benfield.com

Chuck Reynolds

chuckr@benfield.com excerpts adapted from the interviews were used for momentto-moment affect trace analysis in an online survey. Cluster analysis was used to segment gastroenterologists into mutually exclusive provider groups.

Results One hundred and six gastroenterologists completed the survey ( $88 \%$ male; $55 \% \leq 50$ years of age). Over three-fourths of gastroenterologists were familiar with SDM $(77 \%)$. The vast majority of gastroenterologists $(80 \%)$ tried to use a form of SDM with their patients; only $12 \%$ stated that they have a systematic, consistent, and formally documented approach to SDM. Three unique physician clusters were identified: SDM Believers $(20 \%$, $n=20)$; SDM Skeptics ( $47 \%, n=47$ ); and SDM Enthusiasts $(34 \%, n=34)$. The three key barriers to practicing SDM were lack of the following: time (74\%), reimbursement $(70 \%)$, and tools $(51 \%)$. Twenty-two percent of gastroenterologists do not currently use SDM tools.

Conclusions Gastroenterologists lack the systematic approaches and tools for implementing SDM within their IBD

Inflammatory Bowel Disease Center, Dartmouth-Hitchcock Medical Center, Lebanon, NH, USA

2 Janssen Scientific Affairs, LLC, 850 Ridgevew Drive, Horsham, PA 19044, USA

Present Address: Incyte Corporation, Wilmington, DE, USA

4 Delta Modelling, Mt. Prospect, IL, USA

5 BDJ Solutions, Melrose, MA, USA

6 The Benfield Group, St. Louis, MO, USA 
practices. These data offer a foundation for future research in developing and testing SDM programs for gastroenterologists and their IBD patients.

Keywords Shared decision making - Gastroenterology • Colitis ulcerative · Inflammatory bowel diseases · Crohn's disease - Qualitative research

\section{Introduction}

Inflammatory bowel disease (IBD) is a complex disease of the gastrointestinal system and is comprised mainly of two major chronic disorders, Crohn's disease and ulcerative colitis. The chronicity of IBD, characterized by intermittent periods of active disease and remission, requires maintenance therapy usually with a combination of therapeutic agents. Biologic therapies [e.g., anti-tumor necrosis factor (anti-TNF) agents] have become a standard treatment option for patients with IBD, because of their effectiveness in achieving and maintaining IBD remission, particularly in combination with immunomodulator (thiopurine) therapy [1]. However, having a conversation with patients about the complex benefit-risk profiles of these agents is often difficult and is a major cause of patient psychosocial distress in the decision-making process [2].

Research suggests that IBD patients often have only brief and limited conversations about treatment options with their gastroenterologists [3]. Patients with IBD may benefit from a more collaborative and empowering decision-making process that results in a more balanced understanding of therapeutic options, a more satisfying relationship with their physician, and increased treatment satisfaction [4-6].

Shared decision making is a process in which decisions are shared between physicians and patients, informed by the best evidence available, and weighted according to the specific characteristics and values of the patients [7]. Physicians often use decision aids in the shared decisionmaking process to assist patients in making decisions regarding treatment options. Shared decision-making tools increase patient participation in making treatment decisions, reduce conflict regarding treatment plans, and increase patient treatment satisfaction [4-6]. Other potential benefits of the shared decision-making process and use of shared decision-making tools may include a reduction in medical costs, increased physician satisfaction, and improved patient management of disease [8-10].

At least one study has described patients' perspectives on shared decision making [11]. However, to date, there is limited information on gastroenterologists' perspectives of shared decision making or tools used in discussions of therapeutic agents with IBD patients in clinical practice [11]. The objective of the current study was to examine gastroenterologists' perspectives and insights into shared decision making with IBD patients, using a novel statistical hybrid approach to analyze qualitative data. This hybrid research method included moment-to-moment affect trace analyses to evaluate gastroenterologist responses to qualitative audio content.

\section{Materials and Methods}

This was a mixed-methods research study consisting of qualitative and quantitative components. This hybrid approach consisted of the following: (1) a qualitative component-in-depth interviews and (2) a quantitative component - an online physician survey using key in-depth interview insights into moment-to-moment rated discussion.

\section{Qualitative Research}

Ten 30-min in-depth telephone interviews were conducted with 10 physicians familiar with shared decision making in December 2011 (nine gastroenterologists and one plastic surgeon with extensive shared decision making experience). These physicians who were considered experts in their field were identified by one of the authors (CAS) as having experience with and strong opinions about shared decision making (both positive and reserved). During these interviews, physicians were asked questions regarding their experience, concerns, and practicality of shared decision making in order to identify barriers and opportunities to more effectively and efficiently implement shared decision making. The in-depth interview transcripts and digital recordings (provided in online appendix) were reviewed and analyzed to identify differing opinions and physician perspectives expressed during the interviewing process. Excerpts from the transcripts were selected to form an abbreviated script, which was reenacted by actors to protect the anonymity of the physicians actually interviewed. An electronic digital recording of the actors role-playing, based upon the exact words and phrases in the original transcript, was created for use later in moment-to-moment rating of the in-depth physician interviews by the physician participants. The content provided from the in-depth interviews with gastroenterologists was based on interviewees' professional opinions. As such, the statements made by gastroenterologists in the interviews were not particularly emotional in their delivery. Actors voicing the range of opinions expressed provided a more uniform delivery, which allowed participants to rate the content of the opinion rather than the delivery. 


\section{Quantitative Research}

The quantitative research design incorporates two methodologies: a 15-min online quantitative survey among gastroenterologists and a moment-to-moment rating of the actor reenacted excerpts from in-depth physician interviews. In April 2012, 106 gastroenterologists from the Research Now (Plano, TX) Physician Panel opted in to take an 18-question online survey on shared decision making related to IBD. The physicians were asked about the barriers to shared decision making with IBD patients, decision-making practices in IBD, relationship with their IBD patients, knowledge of shared decision making, and insights into shared decision-making implementation in IBD (see survey in online appendix).

An integral part of the online quantitative survey is the moment-to-moment trace rating of the 7-min digital recording based on the physician in-depth interviews. After answering a series of discrete quantitative questions, the 106 physician participants watched a 1-min-long video tutorial that explained the use of a media rating tool and allowed participants to practice using the tool (see online appendix for audio training video script). Physician participants were also asked to "listen and rate" a recording with varying views and opinions about shared decision making and related issues. While online listening to the digital recording, physicians used the mouse or touchpad on a personal computer as an electronic slider moving along a 10-point Likert scale (where $0=$ "completely disagree"; $5=$ "neutral"; $10=$ "completely agree") to rate their agreement or disagreement to what was said throughout the audio recording. After rating audio based on the physician in-depth interviews, the participants were asked a set of discrete follow-up questions related to the topics presented in the audio recording.

\section{Statistical Analyses}

An average of all participants' moment-to-moment ratings/traces was presented as a single "all-trace" line. Additional moment-to-moment trace lines using demographic and other discrete variables such as gender, age, and physician length of practice were used to examine subsegments of the sample.

\section{Cluster Analysis}

$\mathrm{K}$-means cluster analysis was used as a data-mining technique to detect the distinct underlying patterns in media moment-to-moment rating data that could not otherwise be uncovered by looking at traditional demographic or other physician segments. This technique places each participant in a mutually exclusive cluster group having similar patterns of response. Specifically, physician response patterns within a cluster group are more similar to each other than the patterns identified in the other clusters. The consistent pattern of the moment-to-moment traces within each cluster segment is an overall reflection of the group's attitudinal and cognitive response and reaction to the issues put forth in the shared decision-making discussion. The response patterns for each cluster are remarkably different from the "all-trace" or any other trace pattern generated by standard demographics or a priori participant segments. Examining the trace patterns of all the clusters over the course of an entire rated audio discussion visually illustrates where clusters converge on a common point of view (i.e., agree) and where their views dramatically differ (i.e., disagree). There were five physicians rating data that formed an outlier group that was not used in the analysis of clusters.

\section{Segmentations}

Clusters were named based on their respective cognitive responses and attitudes inherent in moment-to-moment trace patterns. Each cluster name was based upon the unique response pattern and reaction (positive/negative) related to the statements in the audio recording. Clusters were profiled using cross-tabulations and the Chi-square test to determine significant differences in the frequency distributions between the clusters with respect to specific demographic variables. Multiple discriminant analysis was used to identify significant moments (points of divergence) between cluster groups in terms disagreement at various points in the discussion. The frequency distribution (percentage of population) was reported for categorical variables, and descriptive statistics (mean, standard deviation) were reported for continuous variables.

\section{Multiple Discriminant Analysis}

Multiple discriminant analysis was used to determine divergent points of discussion between the three clusters. The potency index was calculated based upon the structure matrix and eigenvalue to determine the overall impact of each second of the discussion group. Statistically significant sub-segments (blocks of time in seconds) within the discussion were identified as the most divisive and discriminating opinions between the clusters.

\section{Results}

Of the 106 gastroenterologists who completed the survey, $93(88 \%)$ were male. Over half of gastroenterologists were younger than 50 years of age $(55 \%)$. The age distribution 
was $33,22,32,9$, and $4 \%$ in the 30-39, 40-49, 50-59, $60-69$, and over 70 age groups, respectively. Almost half (46\%) of the gastroenterologists reported working in a private setting, and $34 \%$ reported working in a hospital setting. The majority of gastroenterologists were experienced with $63 \%$ having practiced for 10 years or more and $37 \%$ having practiced for 20 years or more. Less than half of gastroenterologists (42\%) reported seeing 26-75 IBD patients in a month; $37 \%$ reported seeing 10-25 patients in a month.

\section{Attitudes}

Gastroenterologists' attitudes generally aligned in favor of shared decision making. Over three-fourths of gastroenterologists were familiar with shared decision making (between somewhat and extremely familiar-77\%). Physicians had a largely positive view of shared decision making and its benefits (Fig. 1). Over three-fourths of gastroenterologists agreed that shared decision making leads to increased patient satisfaction (strongly agree$55 \%$; somewhat agree-37\%). Over three-fourths of respondents had some agreement that using shared decision making leads to better clinical outcomes (strongly agree$34 \%$; somewhat agree-46\%). Gastroenterologists responded that shared decision making is appropriate in many situations, including "selecting a course of treatment that may have significant risks and benefits" (87\%) and "deciding on elective surgical procedures" (78 \%) (Fig. 2). The vast majority of gastroenterologists $(80 \%)$ responded that they tried to use a form of shared decision making with their patients; however, only $12 \%$ stated that they have a systematic, consistent, and formally documented approach to shared decision making.

\section{Cluster Analyses}

Cluster analyses revealed three physician clusters with unique moment-to-moment trace patterns (Table 1; Fig. 3): Shared decision-making Believers $(19.8 \%, n=20)$; Shared decision-making Skeptics $(46.5 \%, n=47)$; and Shared decision-making Enthusiasts $(33.7 \%, n=34)$. Shared decision-making Believers felt that shared decision making improves patient satisfaction and engages patients, leading to better patient adherence and potentially better outcomes. These gastroenterologists felt that lack of time and lack of payment for services were barriers to shared decision-making implementation. They also believed that shared decision making would provide legal protection and if legal protection is demonstrated, they would have an even greater interest in implementing shared decision making.

Shared decision-making Skeptics were less likely to see patients as qualified to participate in treatment decisions. They had neutral agreement that shared decision making has an impact on patient satisfaction and outcomes. Sixty percent $(60 \%)$ of Skeptics were confident in clinical centers of excellence versus $77 \%$ of Enthusiasts and $90 \%$ of Believers $(p=0.047)$. Similarly, only $19 \%$ of skeptics were confident in the public sector's ability to provide useful shared decision-making tools compared with $44 \%$ of Enthusiasts and $55 \%$ of Believers $(p=0.035)$. In contrast, shared decision-making Enthusiasts believed that physicians should engage patients in the decision-making

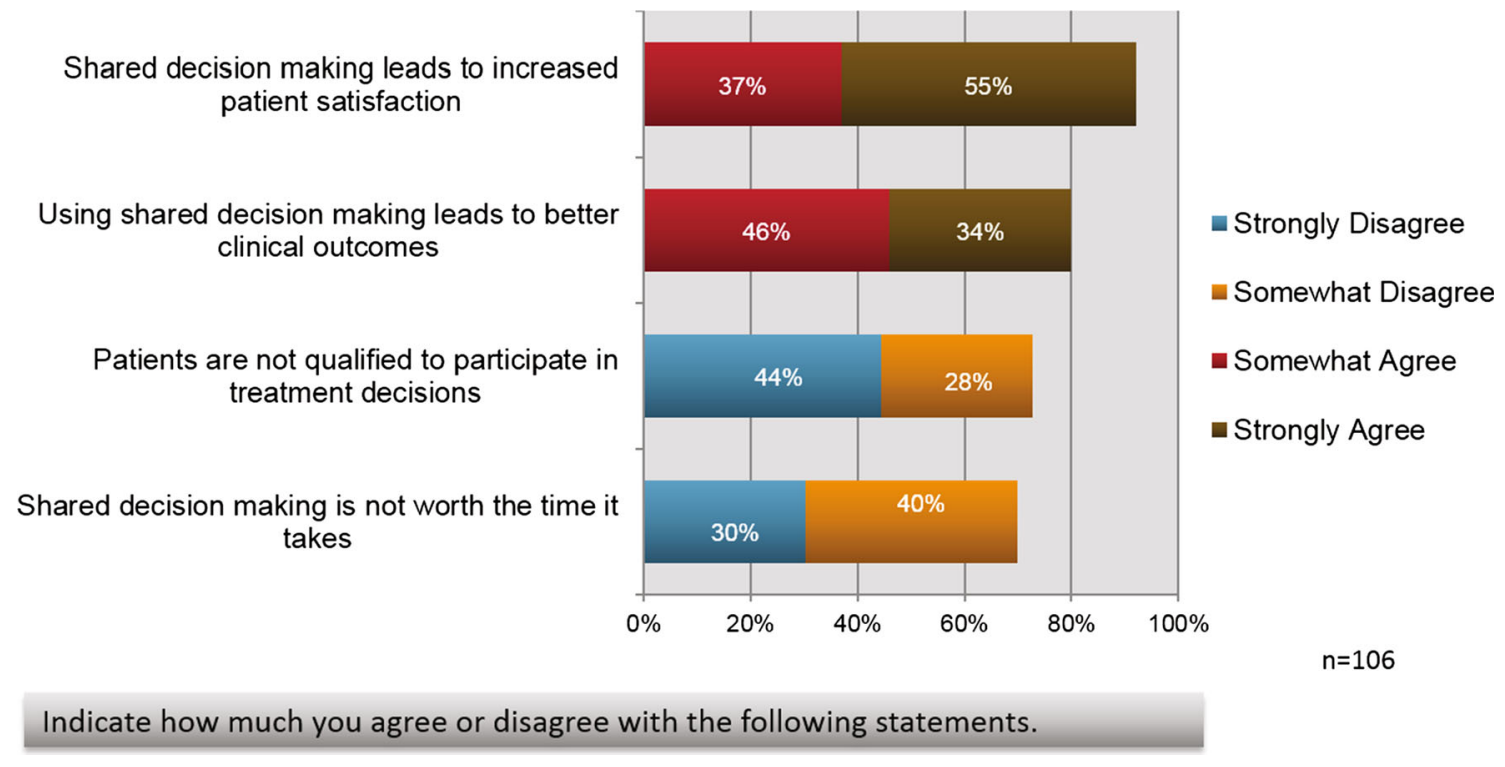

Fig. 1 Gastroenterologists' view of shared decision making and its benefits 


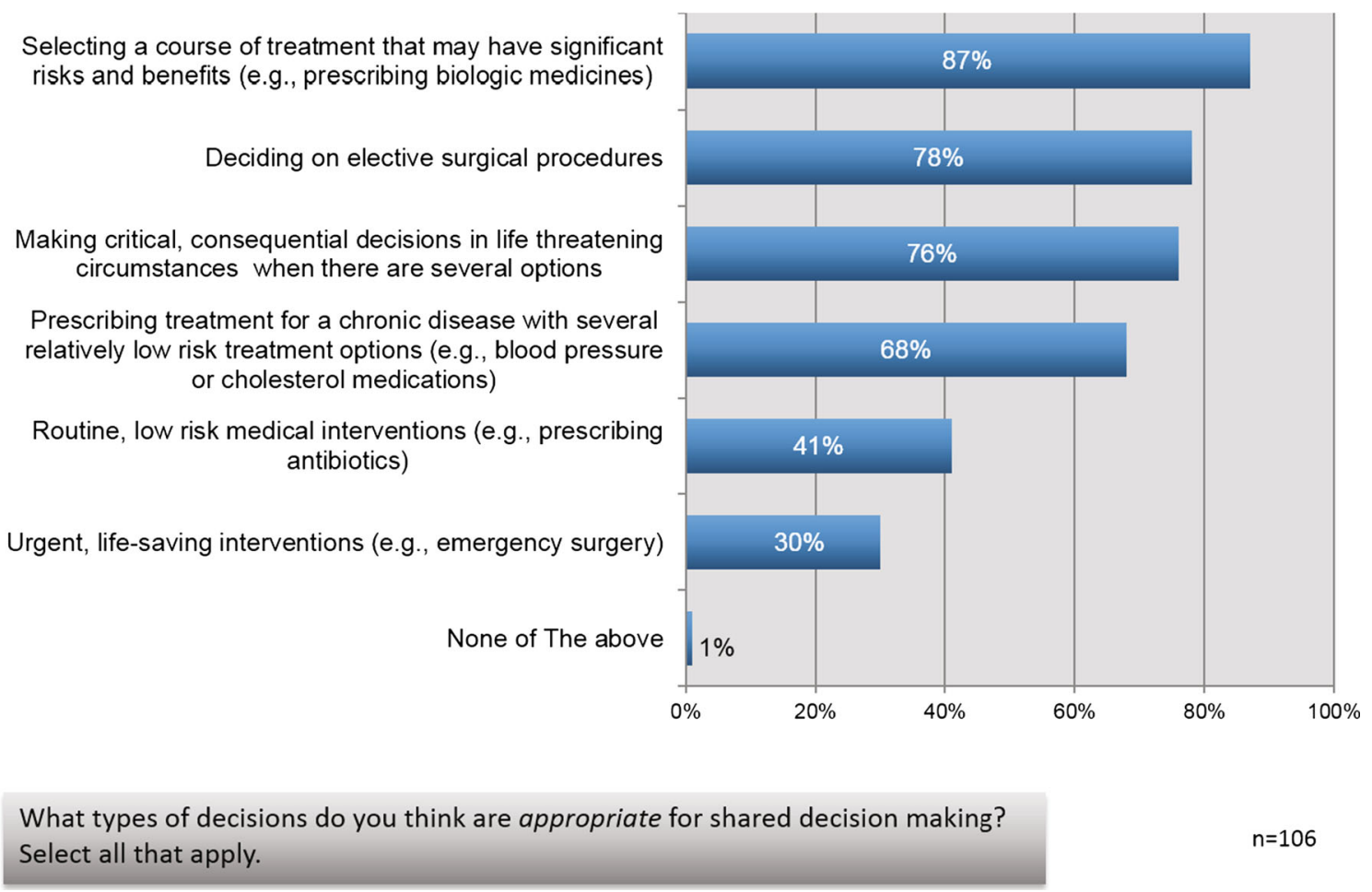

Fig. 2 Gastroenterologists believe shared decision making is appropriate in many situations

Table 1 Gastroenterologist cluster analysis (see Fig. 4 for representative quotes)

\begin{tabular}{|c|c|}
\hline Physician cluster & Characteristics \\
\hline $\begin{array}{l}\text { Shared decision- } \\
\text { making }\end{array}$ & $\begin{array}{l}\text { Believe that shared decision making improves patient satisfaction and engages patients, leading to better patien } \\
\text { adherence and potentially better outcomes }\end{array}$ \\
\hline "Believers" & Feel that time and lack of payment for services are barriers to shared decision-making implementation \\
\hline$(19.8 \%)$ & $\begin{array}{l}\text { Believe shared decision making would provide legal protection } \\
\text { If this is demonstrated, they will have an even greater interest in implementing shared decision making } \\
\text { Are less likely to document shared decision making }\end{array}$ \\
\hline Shared decision- & Are less likely to see patients as qualified to participate in treatment decisions \\
\hline making & Had neutral agreement that shared decision making has an impact on patient satisfaction and outcomes \\
\hline $\begin{array}{l}\text { "Skeptics" } \\
(46.5 \%)\end{array}$ & Process of shared decision making is less systematic and not well documented \\
\hline Shared decision- & Believe that patients' opinions on treatment need to be respected even if a physician disagrees \\
\hline making & Have a strong agreement that shared decision making will have an impact on patient satisfaction and outcomes \\
\hline $\begin{array}{l}\text { "Enthusiasts" } \\
(33.7 \%)\end{array}$ & Most likely to currently use and document shared decision making \\
\hline
\end{tabular}

process and that patient opinions on treatment need to be respected even if a physician disagrees. Enthusiasts had strong agreement that shared decision making would have an impact on patient satisfaction and outcomes and were most likely to currently use and document shared decision making (Fig. 4). Figure 4 depicts a visual representation of moment-to-moment ratings, by physician cluster, of the reenacted in-depth physician interview by physician participants, through the use of a mouse or touchpad on a personal computer to slide along a 10-point Likert scale based on the participant's degree of agreement or disagreement. Of particular note, is the strong disagreement among Enthusiasts with the statements "I don't think shared decision making changes clinical outcomes" and "Just because a patient feels good about his decision, or is confident in his choices, doesn't mean he's making a good decision."

Over half of shared decision-making Believers (55\%) and one-third of Enthusiasts (33\%) were more likely than Skeptics to indicate that they make sure to use shared 


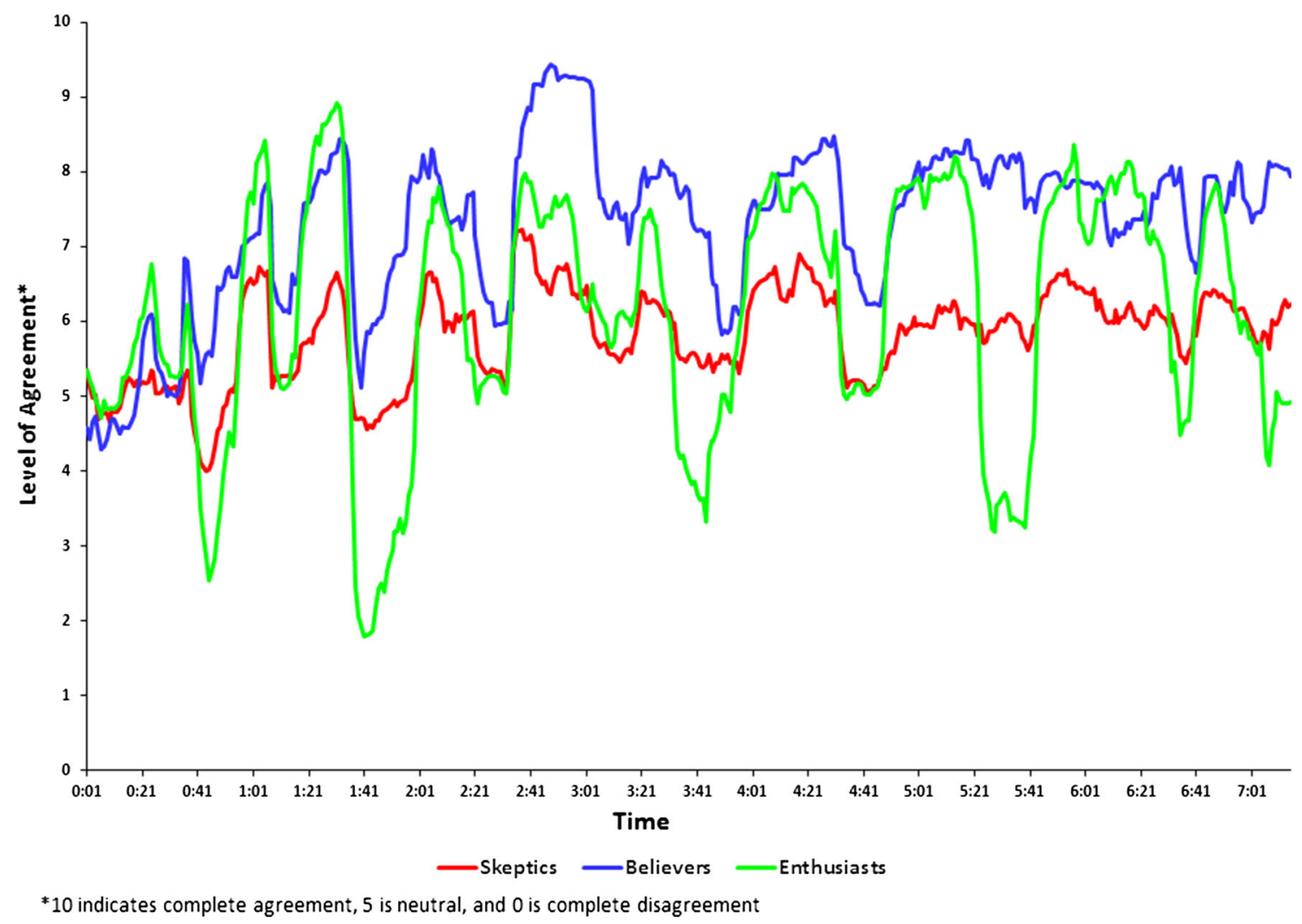

Fig. 3 Moment-to-moment trace patterns by unique physician clusters

decision making with their patients while Skeptics were more likely to indicate that they try to use shared decision making but "don't push it" (77 \%, $p=0.045)$. Over half of shared decision-making Enthusiasts $(58 \%, \mathrm{p}=0.052)$ indicated that their practice of shared decision making is systematic and well documented versus $50 \%$ of Believers and $28 \%$ of Skeptics. In contrast to the physician momentto-moment ratings broken out by cluster, the segment ratings including length of practice (Fig. 5), number of IBD patients per month, and familiarity with shared decision making yielded little insight. These variables were not valuable as a means to discriminate physician views on shared decision making.

\section{Key Barriers}

Gastroenterologists responded that the three key barriers to practicing shared decision making were lack of the following: time (74\%), reimbursement $(70 \%)$, and tools or decision aids $(51 \%)$. Other barriers to practicing shared decision making reported by gastroenterologists included: (1) not enough evidence that shared decision making will produce better clinical outcomes (28\%), (2) fear of legal liability (28\%), (3) not enough evidence that shared decision making will produce increased patient satisfaction
(19\%), and (4) lack of space in their office or practice setting to conduct shared decision making (10\%).

\section{Shared Decision-Making Tools}

The majority of gastroenterologists reported that the most important feature of a shared decision-making tool was one that appealed to patients and was easy to use (75\%). Other important features of a shared decision-making tool included: (1) communicates treatment risk and benefit information in ways patients can understand (59\%), (2) could be used by allied staff to help patients (39\%), (3) could be used at home or in the office (34\%), (4) summarizes information about the patient before their office visit $(33 \%)$, (5) captures information about the patient's fears, values, lifestyles, and lifestyle priorities $(28 \%)$, and (6) comes in multiple formats (e.g., online, DVD, print, smartphone application; $17 \%$ ). Almost three-fourths (72\%) of Skeptics indicated that "communicates treatment risk and benefit in ways patients can understand" is one of the three most essential features in creating a shared decision-making tool compared with $60 \%$ of Believers and $41 \%$ of Enthusiasts $(p=0.019)$.

Twenty-two percent of gastroenterologists responded that they currently do not use shared decision-making tools. 


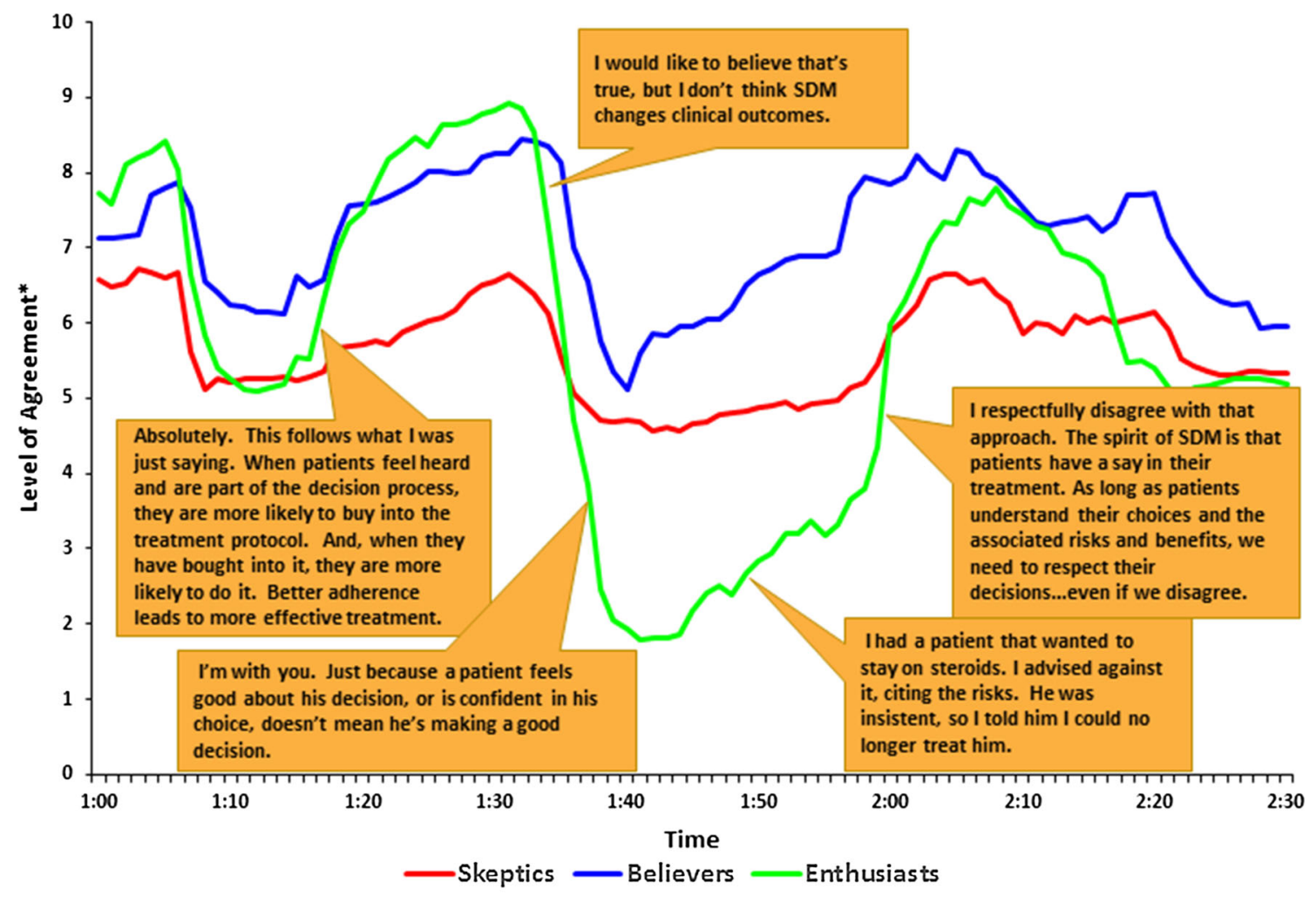

*10 indicates complete agreement, 5 is neutral, and 0 is complete disagreement

Fig. 4 Respondent moment-to-moment responses based on cluster analysis—audio segment: perspectives on shared decision making

Those who responded that they do use tools or decision aids $(70 \%)$ primarily use printed materials such as brochures and copies of articles. Others reported using Web sites $(41 \%)$, worksheets that walk patients through decision factors $(22 \%)$, and videos (7\%). Gastroenterologists have the greatest confidence in professional organizations to provide shared decision-making tools (clinical centers of excellence-72\%; professional society/college-78\%). Respected providers and colleagues with expertise in shared decision making are also a trusted source $(55 \%)$. Over one-third (36\%) of shared decision-making Enthusiasts indicated that they use worksheets with patients compared with $20 \%$ of Believers and $15 \%$ of Skeptics $(p=0.084)$. Similarly, Enthusiasts were more likely to use Web sites as a tool (58\%) compared with approximately $35 \%$ of Skeptics and Believers $(p=0.098)$.

\section{Discussion}

In March 2012, the National Academy for State Health Policy produced a report on shared decision making that advocated the advancement of patient-centered care through federal and state legislation [12]. This report emphasizes the current relevance of implementing shared decision making nationally in alignment with ongoing healthcare reform initiatives. Shared decision-making efforts have been raised as a priority in the Affordable Care Act (ACA), which calls for "certification of patient decision aids; the development, update, and production of patient decision aids to assist providers in educating patients; and, grants to support implementation."

Given the dearth of shared decision-making research among IBD stakeholders and the ripe climate for legislative change, the results of the current study are timely and relevant. The current decision-making approach for IBD treatment in the gastroenterologist-patient conversation has left some patients and caregivers confused and concerned about IBD treatment [2]. Understanding gastroenterologists' perspectives on shared decision making is essential for implementing appropriate tools for individuals with IBD [13]. Furthermore, with the recent availability of shared decision-making tools for IBD, there is an opportunity to develop and test a shared decision-making program for individuals with IBD while meeting the needs of gastroenterologists [13, 14].

Gastroenterologists in the current study identified reasons for resistance to practicing shared decision making. These reasons were described as a lack of the following in clinical practice: time, reimbursement, shared decision- 


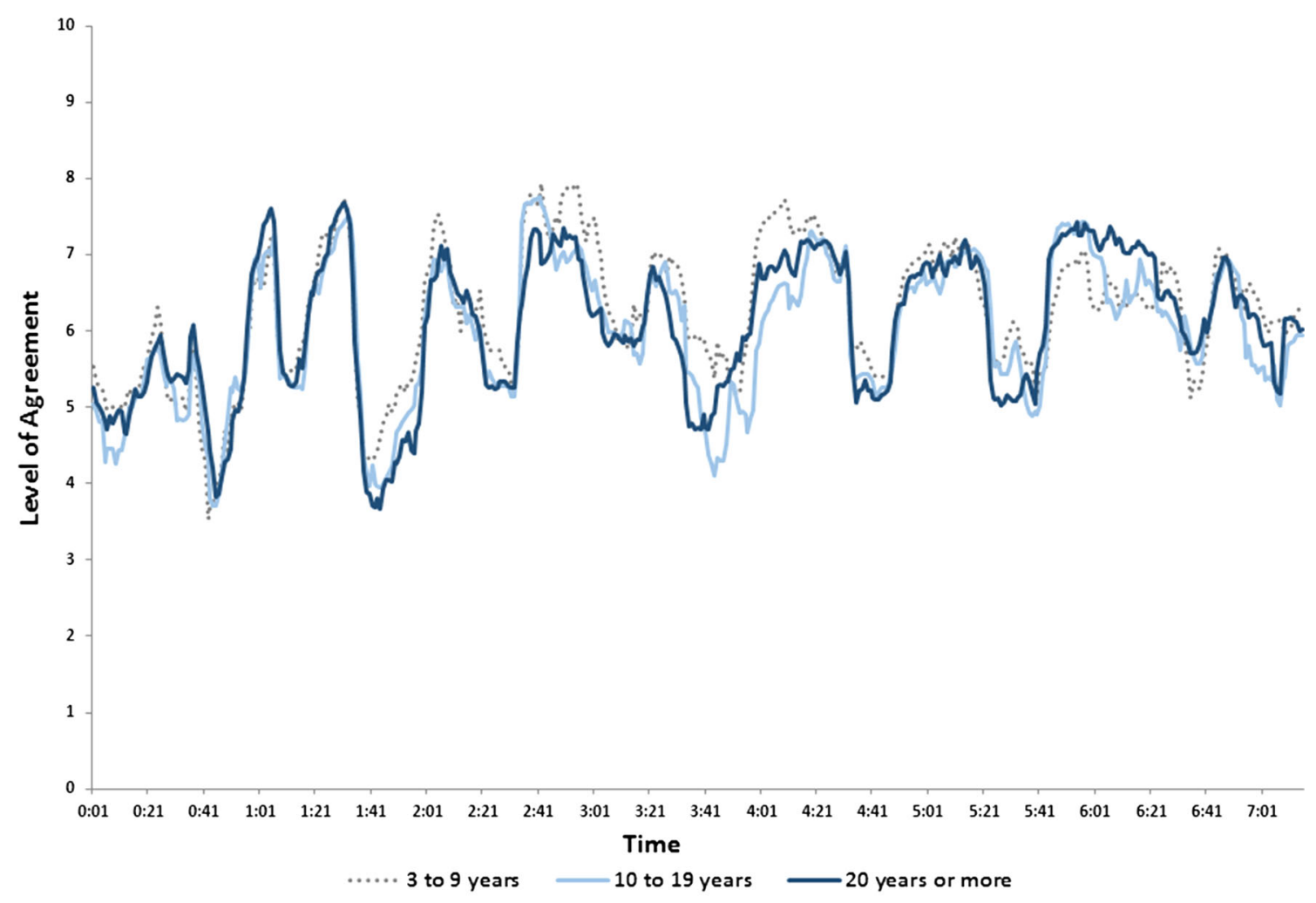

** Note: 6 respondents who practiced "fewer than 3 years" are not included because the $\mathrm{n}$ is too small.

Fig. 5 Moment-to-moment trace patterns by length of physician practice

making tools or decision aids, data supporting better clinical outcomes and patient satisfaction, and office space. These barriers to implementing shared decision making are similar to those reported by other healthcare professionals $[12,15,16]$.

Approximately three-fourths of gastroenterologists in this study stated that they were using shared decisionmaking tools and identified the important features of a shared decision-making tool. They identified their greatest resources as printed materials, Web sites, and professional organizations. Although most of the doctors responded that they are using shared decision-making tools, it is clear that many of the "tools" to which they refer are informational media versus true decision aids. Regardless, use of these educational materials suggests that gastroenterologists are clearly open to engaging in properly developed shared decision-making tools. Studies have shown that the use of shared decision-making tools improves knowledge of healthcare choices, increases the proportion of patients with realistic perceptions of benefits and harms, lowers decisional conflict, reduces the number of patients passively involved in decision making, reduces the number of patients undecided after counseling, improves agreement between patient values and the healthcare option chosen, and reduces costs [8, 17-19]. However, one-fourth of gastroenterologists in this study reported that they do not use shared decision-making tools. One of the reasons given by gastroenterologists in this study for resistance to practicing shared decision making was the lack of IBD decision aids. These data suggest that the availability of an IBD decision aid may facilitate the practice of shared decision making by gastroenterologists in the IBD clinical setting.

The results of this study demonstrated that gastroenterologists are familiar with shared decision making but lack systematic, consistent, and documented approaches and tools for implementing it within their practice settings. Using moment-to-moment rated discussion as a novel methodology for identifying the varying perceptions of shared decision making among gastroenterologists, three unique physician groups were identified: shared decisionmaking Believers (20\%), Skeptics (47 \%), and Enthusiasts (34\%). The larger group of Skeptics confirms the current lack of acceptance and implementation of shared decision making among gastroenterologists in conversations with IBD patients. The main difference between gastroenterologist shared decision-making Believers and Enthusiasts is that Enthusiasts are more likely to systematically document shared decision making and use tools (Web sites and worksheets). Although systematic documentation of shared decision making has not been standardized and 
varies by setting, many organizations have suggested the benefits of documenting the shared decision-making process [20].

Further explanation of the concept of the three physician clusters in the context of moment-to-moment rating studies may be helpful. During the analysis phase of the moment-tomoment results, we compared the ratings of different segments including demographic subgroups and clusters. We identified segment ratings that were nearly identical, distinctly different (e.g., moving in the opposite direction), and different in intensity or degree of ratings. Comparing these segment ratings enabled better understanding of where there was a consensus of opinion and divergence of opinions, and where certain segments felt more or less strongly about a topic than another segment. The difference in degree found in the moment-to-moment ratings is analogous to looking at the rating of a restaurant as a 9 or 10 on a 10-point scale versus a 6 or 7 . Both are positive ratings of the restaurant; however, one group is much more enthusiastic.

The novel hybrid mixed-methods approach used in this study effectively integrated both qualitative and quantitative data to identify the shared decision-making needs and practices of gastroenterologists in the context of IBD treatment options. Qualitative data adapted from the indepth physician interviews on shared decision making generated an online moment-to-moment rated discussion to which gastroenterologists could interact and respond. Gastroenterologists who participated in this online rated discussion were able to integrate both positive and negative contents in forming their overall evaluation of the content. Although this type of research has been used extensively and validated in other fields such as television advertisements and political research, this methodology is new in medical research [21-23]. Moment-to-moment research has recently been conducted in IBD patients to identify patients' perceptions of the shared decisionmaking process in IBD treatment [24]. The combined use of moment-to-moment trace analysis, cluster analyses, and multiple discriminant analysis offers a solution to the historical difficulty of quantifying, analyzing, and interpreting qualitative research. Subsequently, the novelty of this hybrid approach in medical research and with IBD is also a limitation of the study. Given the novelty and small sample size of the study, interpretations may not be generalizable to the larger gastroenterologist population or to all gastroenterologist practices. Further studies using this hybrid methodology to evaluate gastroenterologists' perceptions and practice of shared decision making with IBD patients are warranted.

In summary, this was the first study to use a novel research hybrid methodology integrating both qualitative and quantitative data to identify gastroenterologists' perceptions and practices of shared decision making with IBD patients.
Although gastroenterologists are familiar with and interested in shared decision making, they lack the systematic approaches and tools for implementing shared decision making within their IBD practice settings. These data offer a foundation for future research in developing and testing shared decision-making programs for gastroenterologists and their patients with IBD.

Acknowledgments This study was sponsored by Janssen Scientific Affairs, LLC (Horsham, PA). The authors wish to acknowledge the following individual for their contributions and critical review during the development of this manuscript on behalf of Janssen Scientific Affairs, LLC: Kim Poinsett-Holmes, PharmD of Poinsett Publications, Inc. for medical writing and editorial assistance.

Conflict of interest Corey Siegel has received consulting fees from Abbvie, BiolineRX, Given Imaging, Lilly, Janssen, Salix, Millenium, Pfizer, Prometheus, Takeda, UCB as well as grants from CCFA, AHRQ (1R01HS021747-01) Abbvie, Janssen, Salix, Warner-Chilcott, and UCB. Jennifer Lofland is an employee of Janssen Scientific Affairs, LLC. At the time of the study, Ahmad Naim was an employee of Janssen Scientific Affairs, LLC. Danielle Walls, Jan Collins, Laura Rudder, and Chuck Reynolds were employed as subcontractors by Janssen Scientific Affairs, LLC.

Open Access This article is distributed under the terms of the Creative Commons Attribution-NonCommercial 4.0 International License (http://creativecommons.org/licenses/by-nc/4.0/), which permits any noncommercial use, distribution, and reproduction in any medium, provided you give appropriate credit to the original author(s) and the source, provide a link to the Creative Commons license, and indicate if changes were made.

\section{References}

1. Colombel JF, Sandborn WJ, Reinisch W, et al. Infliximab, azathioprine, or combination therapy for Crohn's disease. $N$ Engl $J$ Med. 2010;362:1383-1395.

2. Lipstein EA, Lovell DJ, Denson LA, et al. Parents' information needs in Tumor Necrosis Factor- $\alpha$ inhibitor treatment decision. $J$ Pediatr Gastroenterol Nutr. 2013;56:244-250. doi:10.1097/ MPG.0b013e31827496c3.

3. Baars JE, Siegel CA, Kuipers EJ, van der Woude CJ. Patient's perspectives important for early anti-tumor necrosis factor treatment in inflammatory bowel disease. Digestion. 2009;79:30-35.

4. O'Connor AM, Bennet CL, Stacey D, et al. Decision aids for people facing health treatment or screening decisions. Cochrane Database Syst Rev 2009; Jul 8: CD001431.

5. Glass KE, Wills CE, Holloman C, et al. Shared decision making and other variables as correlates of satisfaction with health care decisions in a United States national survey. Patient Educ Couns. 2012;88:100-105.

6. Lim E. Patients' perspective in the surgical decision-making process. Thorac Surg Clin. 2012;22:539-543.

7. Towle A, Godolphin W. Framework for teaching and learning informed shared decision making. BMJ. 1999;319:766-771.

8. Kennedy ADM, Sculpher MJ, Coulter A, et al. Effects of decision aids for menorrhagia on treatment choices, health outcomes, and costs. JAMA. 2002;288:2701-2709.

9. Sullivan MD, Leigh J, Gaster B. Brief report: training internists in shared decision making about chronic opioid treatment for noncancer pain. J Gen Intern Med. 2006;21:360-362. 
10. Sieber W, Newsome A, Lillie D. Promoting self-management in diabetes: efficacy of a collaborative care approach. Fam Syst Health. 2012;30:322-329. doi:10.1037/a0030714.

11. Baars JE, Markus T, Kuipers EJ, van der Woude CJ. Patients' preferences regarding shared decision-making in the treatment of inflammatory bowel disease: results from a patient-empowerment stud. Digestion. 2010;81:113-119.

12. Shafir A, Rosenthal J. Shared decision making: advancing patient-centered care through State and Federal implementation. National Academy for State Health Policy. Informed Medical Decisions Foundation. March 2012. http://www.nashp.org/pub lication/shared-decisionmaking-advancing-patient-centered-carethrough-state-and-federal. Last accessed February 24, 2014

13. Siegel CA, Siegel LS, Hyams JS, et al. Real-time tool to display the predicted disease course and treatment response for children with Crohn's disease. Inflamm Bow Dis. 2011;17:30-38.

14. Siegel CA. Review article: explaining risks of inflammatory bowel disease therapy to patients. Aliment Pharmacol Ther. 2011;33:23-32.

15. Légaré F, Ratté S, Gravel K, Graham ID. Barriers and facilitators to implementing shared decision-making in clinical practice: update of a systematic review of health professionals' perceptions. Patient Educ Couns. 2008;73:526-535.

16. Gravel K, Légaré F, Graham ID. Barriers and facilitators to implementing shared decision-making in clinical practice: a systematic review of health professionals' perceptions. Implement Sci. 2006;1:16.

17. Stacey D, Bennett CL, Barry MJ, et al. Decision aids for people facing health treatment or screening decisions. Cochrane Database Syst Rev 2011;10:CD001431.
18. Volandes AE, Barry MJ, Chang Y, Paasche-Orlow MK. Improving decision making at the end of life with video images. Med Decis Making. 2010;30:29-34.

19. Volandes AE, Paasche-Orlow MK, Barry MJ, et al. Video decision support tool for advance care planning in dementia: randomized controlled trial. BMJ. 2009;338:b2159.

20. Moulton B, King JS. Aligning ethics with medical decisionmaking: the quest for informed patient choice. J Law Med Ethics. 2010;38:85-97.

21. Baumgartner H, Sujan M, Padgett D. Patterns of affective reactions to advertisements: the integration of moment-to-moment responses into overall judgments. J Mark Res. 1997;34:219-232.

22. Elpers JLCMW, Wedel M, Pieters RGM. The influence of moment-to-moment pleasantness and informativeness on zapping TV commercials: a functional data and survival analysis approach. In: Broniarczyk SM, Nakamoto K, eds. Advances in consumer research. Valdosta, GA: Association for Consumer Research; 2002.

23. Hughes GD, Lennox RD. The reliability and validity of realtime responses measures. In: Bearden $\mathrm{W}$, et al., eds. Realtime response research: construct validation and reliability assessment. Chicago: American Marketing Association; 1990.

24. Siegel CA, Lofland JH, Naim A, et al. Novel statistical approach to determine inflammatory bowel disease patients' perspectives on shared decision making. Patient. 2015 (in press). 